\title{
Téoros
}

Revue de recherche en tourisme

\section{Bilan de santé et prévisions de l'hôtellerie à Montréal, 1977-1995}

\section{Jean Stafford}

Volume 10, numéro 3, novembre 1991

Destination Montréal : d'hier à demain

URI : https://id.erudit.org/iderudit/1079197ar

DOI : https://doi.org/10.7202/1079197ar

Aller au sommaire du numéro

Éditeur(s)

Université du Québec à Montréal

ISSN

0712-8657 (imprimé)

1923-2705 (numérique)

Découvrir la revue

Citer cet article

Stafford, J. (1991). Bilan de santé et prévisions de l'hôtellerie à Montréal,

1977-1995. Téoros, 10(3), 13-18. https://doi.org/10.7202/1079197ar d'utilisation que vous pouvez consulter en ligne.

https://apropos.erudit.org/fr/usagers/politique-dutilisation/ 


\section{Bilan de santé et prévisions de l'hôtellerie à Montréal, 1977-1995}

\section{Jean Stafford"}

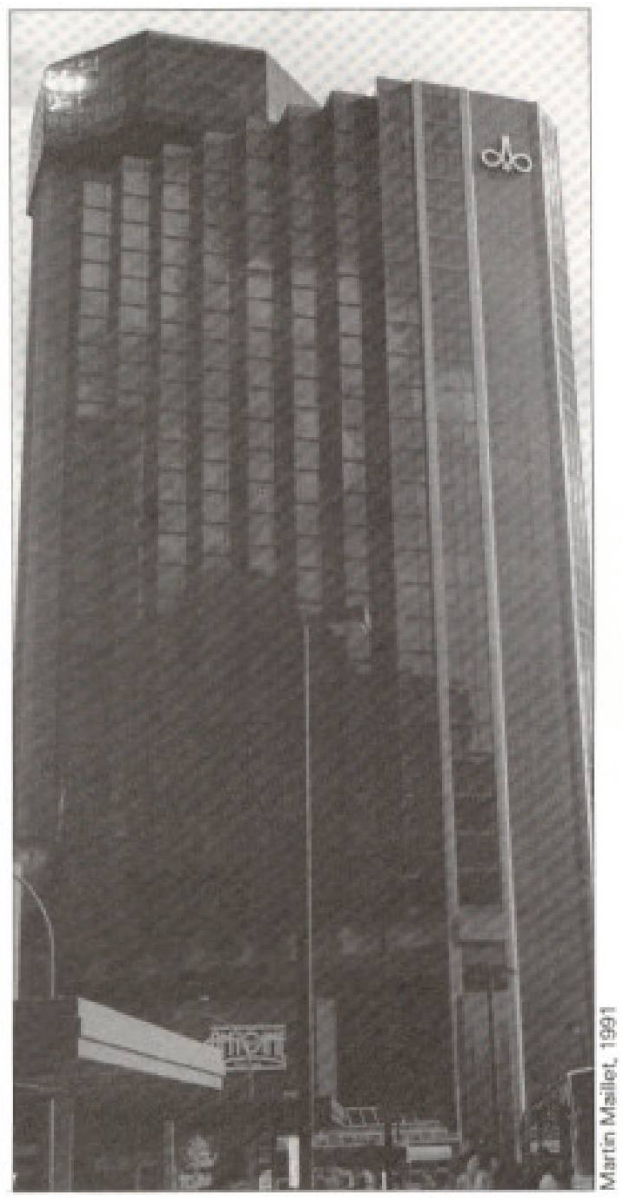

Dans une perspective à long terme, lindustrie hôtelière semble bien se porter malgré des forces contraires.

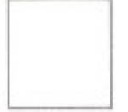

L'hồtellerie joue un rôlle bien spécifique dans la fonction d'accueil de la région de Montréal. L'hébergement sert de support à l'ensemble des activités économiques et socio-culturelles (ces deux activités étant fortement reliées). L'hôtellerie sert en fait d'infrastructure à tout le système d'échanges nationaux et internationaux caractéristique d'une métropole. L'hôtellerie entre ainsi en symbiose avec un secteur des services très diversifié, avec un secteur financier et commercial offrant une gamme étendue de possibilités d'êchanges.

L'hôtellerie est donc un élément important de la croissance d'une ville, son niveau de santé et un indice de son dynamisme et de l'insertion de cette ville dans les échanges internationaux. Il fautpercevoir l'hôtellerie à Montréal comme un être vivant qui s'adapte à des conjonctures diverses. Si l'hôtellerie peut être perçue comme la vitrine économique de Montréal, ce secteur n'est pas exempt des accès de fièvre propres à tous les marchés économiques.

Ces diverses fluctuations liées aux crises économiques et aux changements dans les comportements des consommateurs (nouvelles perceptions des loisirs touristiques) structurent le marché de l'hébergement. Seule une étude attentive des différents mouvements propres aux séries temporelles de l'hôttellerie montréalaise permettront de tracer un bilan de santé dans ce secteur.

Nous nous proposons donc d'analyser les diverses fluctuations de l'hôtellerie dans les dix dernières années en fonction des différents mouvements (tendanciels, cycliques, saisonniers, irréguliers) et de faire des prévisions à court terme et à moyen terme. Cette analyse se fera au plan macroéconomique en tenant compte surtout de la grande région de Montréal.

\section{Méthodes de la prévision}

Monsieur Jean Stafford est professeur au Departement d'étudesurbaines et touristiques de I'Universite du Québec à Montréal.
Les données utilisées pour les prévisions regroupent l'ensemble des hôtels du Grand
Montréal et selon les délimitations territoriales et les sources qu'utilise l'Association des hôtels du Grand Montréal. Dans cette étude, nous avons privilégié un modele d'ajustement à un modele explicatif. Les séries temporelles peuvent se résumer par la formule suivante:

$$
\begin{aligned}
\mathrm{Y} & =\mathrm{f}(\mathrm{T}, \mathrm{C}, \mathrm{S}, \mathrm{D}) \text {; où: } \\
\mathrm{Y} & =\text { variable à prévoir; } \\
\mathrm{T} & =\text { tendance gênérale de la série; } \\
\mathrm{C} & =\text { mouvement cyclique; } \\
\mathrm{S} & =\text { mouvement saisonnier; } \\
\mathrm{I} & =\text { mouvement irrégulier. }
\end{aligned}
$$

Les valeurs prévues vont done tenir compte des formes spécifiquesque prennent chacun de ces mouvements dans le passé et dans le présent. Ce type de modèle est très souvent utilisé pour les prévisions dans l'industrie touristique ${ }^{(1)}$.

Les mouvements saisonniers sont captés par l'algorithme X11 mis au point par le Bureau de recensement des États-Unis. Selon M. Davidet J.-C. Michaud, ce modèle: «... applique différentes moyennes mobiles à la série afin de dégager la tendance et le facteur saisonnier. Les valeurs aberrantes de la composante résiduelle sontéliminées à chaque étape de la procédure de façon à obtenir des estimations suffisamment lissées des composantes» ${ }^{(2)}$.

Les prévisions à court terme sont obtenues à partir de $1^{4} u n e$ des cinq méthodes prévisionnelles suivantes: lissage exponentiel, Winters, Stepar, X11, X11 et Stepar; ces approches tiennent compte de trois types possibles de tendance (constante, linéaire et quadratique) ${ }^{(3)}$. Pour chacune des séries temporelles (des chambres, des nuitées et des taux d'occupation) nous avons donc quinze séries prévisionnelles et certains tests statistiques nous permettent de choisir la meilleure tendance pour la prochaine année $e^{(4)}$.

La construction des indices cycliques s'est faite à partir de différentes formes de lissage: 
- par des tendances mathematiques linếaires ou quadratiques

(par années);

- par diverses moyennes mobiles (doubles ou triples).

Dans tous les cas, la formule utilisce est:

$\left.\mathrm{ICY}=\frac{(\mathrm{yi}}{\mathrm{Ypr}_{\mathrm{pr}}} \times 100\right)$; où:

$\mathrm{ICY}=$ indice cyclique;

$y_{1}=\quad$ valeurs observées;

$Y \mathrm{pr}=$ valeurs prévues par un modèle quelconque.

Enfin, les prévisions à moyen terme sont calculées à partir de tendances mathêmatiques; à ce moment, les équations mathé matiques les plus utilisés sont:

1- la tendance linéaire: $\mathrm{Y}=\mathrm{b}_{0}+\mathrm{b}_{1}(\mathrm{t})+\mathrm{E}$;

2- la tendance logarithmique

(soit semi-log ou double $\log$ );

$\mathrm{Y}=\mathrm{b}_{\mathrm{g}} \mathrm{X}^{\mathrm{the}}+\mathrm{E}$ et

$L_{\mathrm{a}} \mathrm{Y}=\mathrm{b}_{\mathrm{0}}+\mathrm{b}_{1}(\mathrm{t})+\mathrm{E}$;

3- la tendance polynomiale:

$\mathrm{Y}=\mathrm{b}_{0}+\mathrm{b}_{1}(\mathrm{t})+\mathrm{b}_{2}\left(\mathrm{t}^{2}\right)+\mathrm{E} ;$

4- la tendance inverse:

$\mathrm{Y}=\mathrm{b}_{0}+\underline{\mathrm{b}}_{\mathrm{t}}+\mathrm{E}$.

Comme pour la prévision à court terme, il s'agit, dans ce dernier cas, de choisir la wmeilleure tendances à partir des tests statistiques usuels. Pour terminer, il faut souligner que les prévisions obtenues ne sont qu'une construction therorique de la réalité observée.

\section{L'évolution passée (1980-1990)}

L'étude de la dernière décennie(1980-1990) est importante pourcomprendre laprochaine décennic (1991-2000). L'évolution indicielledes chambres (voir tableau 1) indique une baisse en 1981 et ensuite en 1985; de 1986 a 1990 , on constate une hausse graduelle et relativement importante du nombre des chambres disponibles à Montréal. Le taux d"accroissement annuel moyen (moyenne géométrique) a été de $3,5 \%$ en 1986-1990 par rapport à $0,9 \%$ en 19811985. On remarque aussi que la plus forte baisse se situe en 1981 et $1984(-3 \%)$ et la plus forte hausse en 1987 (6\%).

\section{GRAPHIQUE 1}

Evolution indicielle (base $100=1980$ ) des chambres disponibles et des nuitées à Montréal de 1980 à 1990

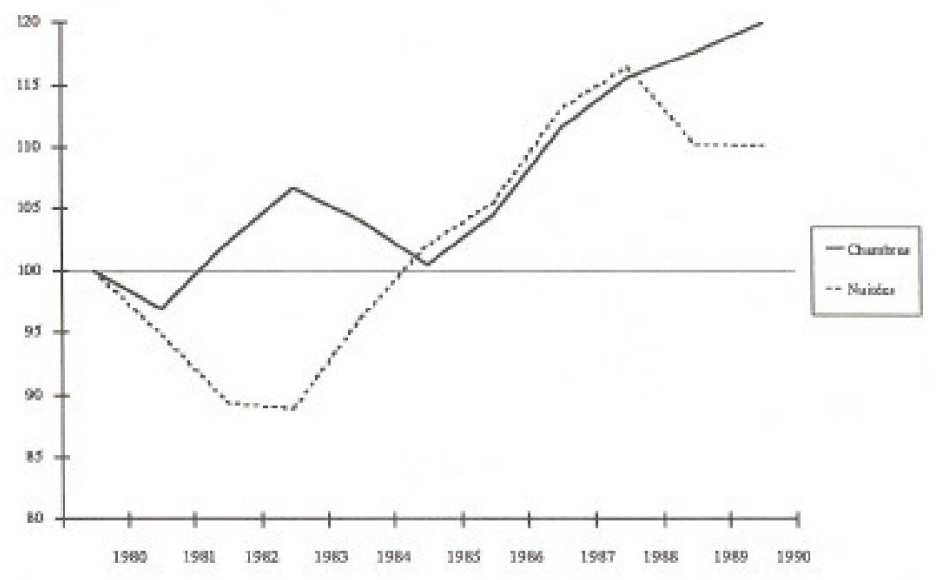

GRAPHIQUE 2

Évolution des indices cycliques des chambres disponibles et des nuitées à Montréal de 1977 à 1990

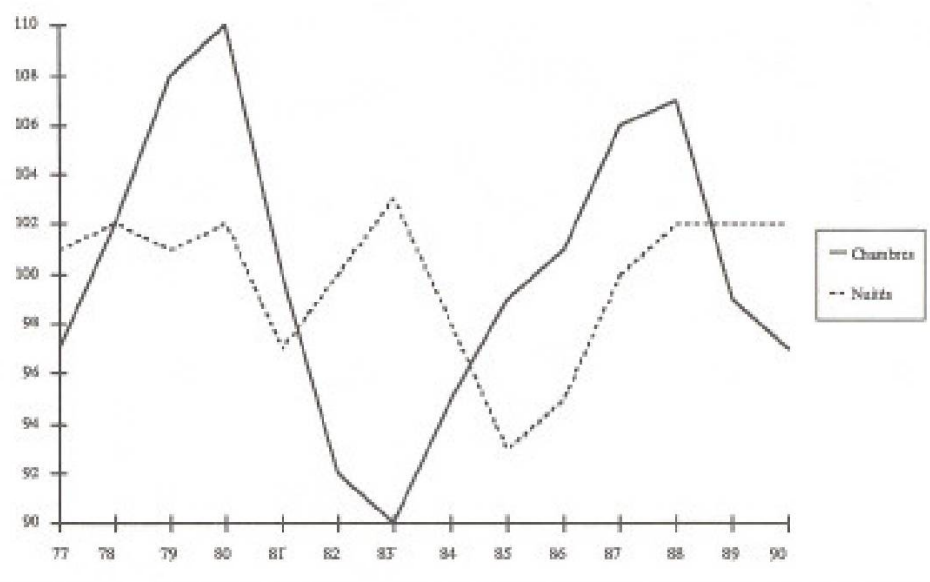

Par rapport à l'année de base (1980), les nuites ont connu une baisse de 1981 à 1984; ensuite, la progression a été constante jusqu'en 1988. Il y a une stagnation des nuitées en 1989 et 1990 . La plus forte baisse des nuitées se retrouve (pour les taux d'accroissement annuel') en $1982(-6 \%)$ et la plus forte hausse en $1987(7 \%)$. Le taux d'accroissement annuel moyen (moyenne géométrique) des nuitées a été de $1,9 \%$ de 1981 à 1985 et de $1,1 \%$ de 1986 à 1990.

Le graphique 1 montre bien $1^{\prime}$ évolution indicielle des deux serries. On peut voir un mouvement inverse entre 1981 et 1985; par la suite, l'ajustement semble se faire de 1985 à 1988. À partir de 1988, les deux courbes se distendent et l'ajustement entre les deux séries ne se fait plus. Même si les deux séries temporelles des chambres et des nuitées évoluent un peu en dents de scie, les coefficients de variation de l'uneet l'autre des séries restent à un niveau acceptDe 1977 à 1990, la moyenne des taux d'occupation est de $64,9 \%$, avec un écart type de $3,9 \%$. La valeur la plus faible observée pendant cette période (annuellement) a été de 57 et la valeur la plus élevée a êté de 69. Le coefficient de variation du taux d'occupation est de $6,1 \%$ de 1977 a 1990.

Le bilan de santé pour l'hôtellerie à Montréal, et pour les périodes considếrées, n'est. 
TABLEAU 1

Évolution indiclelle et taux d'accroissement annuel des moyennes annuelles des chambres disponibles et des nuitées des hôtels de Montréal de 1980 à 1990

\begin{tabular}{lccrrrr}
\hline Années & \multicolumn{3}{c}{ Chambres } & Nuitées \\
\hline & Nombre & $\begin{array}{c}\text { Base } 100= \\
1980\end{array}$ & $\begin{array}{c}\text { TAA } \\
\text { en \% }\end{array}$ & Nombre & $\begin{array}{c}\text { Base 100 } \\
1980\end{array}$ & $\begin{array}{c}\text { TAA }^{*} \\
\text { en \% }\end{array}$ \\
\hline & & & & & & \\
1980 & 332642 & 100 & 3 & 227059 & 100 & 3 \\
1981 & 322260 & 97 & -3 & 215072 & 95 & -5 \\
1982 & 339864 & 102 & 5 & 202855 & 89 & -6 \\
1983 & 354728 & 107 & 4 & 201946 & 89 & -0.4 \\
1984 & 346268 & 104 & -2 & 218561 & 96 & 8 \\
1985 & 334080 & 100 & -3 & 231868 & 102 & 6 \\
1986 & 347842 & 104 & 4 & 239654 & 105 & 3 \\
1987 & 370745 & 111 & 6 & 256905 & 113 & 7 \\
1988 & 384427 & 115 & 4 & 264339 & 116 & 3 \\
1989 & 390984 & 117 & 2 & 250342 & 110 & -5 \\
1990 & 398641 & 120 & 2 & 249926 & 110 & -0.2 \\
\hline
\end{tabular}

* Taux d'accroissement annuel.

TABLEAU 2

Les moyennes des coefficients* saisonniers des chambres disponibles, des nuitées et des taux d'occupation à Montréal de 1977 à 1990

\begin{tabular}{|c|c|c|c|c|c|c|c|c|c|c|c|c|}
\hline Mois & Jan. & Fêv. & Mars & Avril & Mai & Juin & Juil. & Août & Sept. & Oct. & Nov. & Déc. \\
\hline \multicolumn{13}{|l|}{$\begin{array}{l}\text { Indica= } \\
\text { teurs }\end{array}$} \\
\hline Nuitées & 76 & 84 & 90 & 91 & 110 & 112 & 111 & 130 & 124 & 114 & 92 & 65 \\
\hline Taux & 76 & 90 & 89 & 92 & 108 & 114 & 109 & 127 & 125 & 112 & 93 & 64 \\
\hline
\end{tabular}

* Calculés par la méthode X11 de SAS.

\section{TABLEAU 3}

Les moyennes des coefficients salsonniers* des taux d'occupation des hötels à Montréal selon trois pẻriodes: $1977-1984,1985-1990$ et $1977-1990$

\begin{tabular}{|c|c|c|c|c|c|c|c|c|c|c|c|c|}
\hline Mois & Jan. & Fêv. & Mars & Avril & Mai & Juin & Juil. & Aoủt & Sept. & Oct. & Nov. & Déc. \\
\hline \multicolumn{13}{|l|}{ Années } \\
\hline $1977-1984$ & 78 & 91 & 90 & 91 & 105 & 112 & 108 & 128 & 126 & 114 & 93 & 64 \\
\hline $1985-1990$ & 74 & 89 & 91 & 93 & 110 & 114 & 109 & 128 & 125 & 110 & 92 & 64 \\
\hline $1977-1990$ & 76 & 90 & 89 & 92 & 108 & 114 & 109 & 128 & 125 & 112 & 93 & 64 \\
\hline
\end{tabular}

* Calculés par la méthode X11 de SAS. 
ni rose ni noir; il est tout en demie-teintes. Il y a un constant ajustement (avec retard) du nombre des chambres et du nombre des nuitées; dans ce sens, Ie taux d'occupation demeure un bon indicateur de l'évolution đu marché de l'industrie hôtelière à Montréal.

\section{Le mouvement saisonnier}

Le coefficient saisonnier est le meilleur outil pour capter la saisonnalité et lui donner une forme analysable. Les coefficients saisonniers correspondent à un modele thérorique de la saisonnalité(5). L'indice du coefficient saisonnier se lit de la façon suivante: la valeur 100 correspond à la moyenthe annuelle de la variable; les valeurs superieures à 100 indique une hausse par rapport à cette valeur moyenne; les valeurs inféricures à 100 indique une baisse par rapport à cette valeur moyenne.

Le tableau 2 montre bien, qu'en ce qui a trait aux chambres disponibles, il y a peu de variation selon les mois de l'annce. La valeur la plus faible est en fevrier (92) ce qui indique qu'en février, il y a $8 \%$ de moins de chambres disponibles que la moyenne annuelle. Les valeurs les plus hautes (102) sont en juillet-août. Les fluctuations sont faibles car les indices de decembre et janvier sont à 101 .

Par contre, les nuitées connaissent d'importantes fluctuations qui correspon= dent assez bien aux effets de la saisonnalité sur le tourisme. C'esten décembre que l'on retrouve la valeur la plus faible (avec 65); il y a donc $35 \%$ de moins de nuitées que la moyenne annuelle en decembre. A l'autre extrême, il y a le mois d'aoüt avec un coefficientsaisonnier de 130 . Il est curieux d'observer que les mois de septembre et octobre (avec 124 et 114) ont une meilleure performance que les mois de mai, juin et même juillet.

Les coefficients saisonniers des taux d'occupation suiventd'assez près ceux des nuitées. Le tablea 3 montre, que même sur une période longue, les coefficients saisonniers ne comnaissent que de faibles variations. On remarque des changements positifs (valeurs supéricures à la période précédente) de mars a juillet et négatifs (valeurs inférieures à la période précédente) en octobre et novembre ainsi que pour les mois de janvier et février.
Dans l'ensemble, la saisonnalité apeu variée dàs la demiere décennie; elle constitue une forme $\mathrm{d}$ 'invariance sur laquelle nous pouvons compter pour faire des prévisions plausibles. On peut facilement penser que lasaisonnalité influencera encore longtemps ledestin de l'industrie hôtelière à Montróal.

\section{Le mouvement cyclique}

Les fluctuations économiques ont beaucoup d'influence sur l'industrie hôtclière montréalaise. Dans le tableau 4, nous voyons des indicateurs cycliques calculés de façon empirique. On constate, pour les chambres disponibles, une baisse en 1981 et ensuite pour la période 1984 à 1986. Pour cette séric, les variations cycliques sont relativement faibles (le coefficient de variation est de 3\%); la valeur la plus faible est de 93 et la valeur la plus élevée est 103.

Pour les nuitées, les périodes de baisse se situenten 1977, de 1982 à 1985 etenfin, en 1989 et 1990 . Le coefficient de variation est de $5,9 \%$. La valeur la plus faible est 90 et la valeur la plus élevée est 110. $\mathrm{L}^{+}$evolution des indices cycliques des taux d'occupation se rapproche assez de celui des nuitées.

Le graphique 2 montre assez bien que les indices cycliques des chambres et les indices cycliques des nuités 6 voluent de façon assez différente. Dans la periode 1977 a 1980, les indices cycliques des nuitées augmentent alors que les indices cycliques des chambres restent stables ou diminuent (par rapport à la valeur 100). De 1981 à 1984 atde 1984 à 1985 , ainsi qu'en 1989 et en 1990 , les indices cycliques des deux variables ont des comportements completement inversés. Par contre, de 1985 à 1988, les indices cycliques des chambreset des nuitées progressent de concert.

Pour les périodes plus récentes: $1989 \mathrm{et}$ 1990, on remarque que les nuitées sont entróes dans un cycle descendant alors que les indices cycliques des chambres semblent se stabiliser. Nous pouvons faire plusieurs hypothèses pour 1991 at 1992: le cycle des nuitées peutcontinuer à se creuser et le cycle des chambres peut lui aussi faire un plongeon en bas de 100 pour s"arrêter à l'indice 95 ou 96: il est possible aussi que la reprise reprenne vraiment..

\section{Les prévisions à court terme}

Les prévisions à court terme apparaissent dans le tableau 5 ; il s'agit d'une tendance parabolique qui tient compte de la saisonnalité ainsi que des mouvements cycliques et des mouvements irréguliers. Le modèle à court terme prévoit une croissance de $3,6 \%$ deschambres disponibles (en moyenne) pour les douze prochains mois.

Les taux de croissance les plus faibles sont en septembre 1991 (par rapport à septembre 1990) avec $0,05 \%$; de decembre 1990 a décembre 1991, la croissance devrait être de 2,9\%; ensuite viennent: juin 1992 avec $3,5 \%$, juillet 1992 avec $2,3 \%$ et enfin août 1992 avec $1,5 \%$ (en comparaison avec les mêmes mois de 1991).

Les taux de croissance les plusélevés, pour les chambres disponibles sonten mai 1992 , avec $5,9 \%$, févricr 1992 avec $4,8 \%$ et mars 1992 avec $4,6 \%$ (en comparant avec l'anné 1991). L'offre de chambres disponibles devrait donc continuer à augmenter pour les prochains mois de 1991 et ensuite jusqu'en août 1992.

La prévision à court terme des nuités montre une baisse des nuitétes de septembre à décembre 1991 (en comparaison avec les mêmes mois de 1990). La baisse des nuitées prévues est importante car la moyenne pour cesquatre moisest de $-5,5 \%$ (avec $-9,9 \%$ en septembre $1991 \mathrm{et}-6 \%$ en novembre 1991).

De janvier à août 1992, les nuitées devraient s'accroitre; le taux moyen de croissance pour ces périodes serait de $6,8 \%$. On rèmarque une augmentation importante de $9,4 \%$ en février 1992 , de $15,3 \%$ en mars 1992 et de $7,9 \%$ en juillet 1992 (par rapport aux mêmes mois de 1991).

Les taux d'occupation, selon le modele prévisionnel, diminueraient (par rapport à 1990 ) de septembre 1991 a janvier 1992. Les taux d'occupation augmenteraient de février à avril $1992 \mathrm{et}$ ils diminueraient à nouveau en mai et juin 1992; pour les mois de juilletet août 1992, les taux d'occupation seraient en croissance par rapport aux mêmes mois de 1991.

Dans l'ensemble, les prévisions à court terme ne sont guère reluisantes: le modele prevoit une augmentation des chambres disponibles et un taux de croissance plus faible des nuités, ce qui faitque les derniers mois de 1991 et les huit premiers de 1992 


\section{TABLEAU 4}

Indices cycliques* des chambres disponibles, des nuitées et des taux d'occupation à Montréal de 1977 à 1990

\begin{tabular}{rrrr}
\hline Années & Chambres & Nuitées & Taux \\
\hline 1977 & 101 & 97 & 97 \\
1978 & 102 & 102 & 98 \\
1979 & 101 & 108 & 106 \\
1980 & 102 & 110 & 106 \\
1981 & 97 & 100 & 103 \\
1982 & 100 & 92 & 90 \\
1983 & 103 & 90 & 88 \\
1984 & 98 & 95 & 97 \\
1985 & 93 & 99 & 106 \\
1986 & 95 & 101 & 106 \\
1987 & 100 & 106 & 104 \\
1988 & 102 & 107 & 104 \\
1989 & 102 & 99 & 97 \\
1990 & 102 & 97 & 95 \\
\hline
\end{tabular}

* Calculés à partir des moyenes annuelles (voir tableau 1).

\section{TABLEAU 6}

Les prévisions* de la moyenne annuelle des chambres disponibles dans les hôtels de Montréal, de 1991 à 1995, en fonction de quatre hypothèses d'évolution de la conjoncture économique

\begin{tabular}{|c|c|c|c|c|}
\hline $\begin{array}{l}\text { Hypothèses } \\
\text { Annëes }\end{array}$ & ses & B & $\mathrm{C}$ & D \\
\hline & $\begin{array}{c}\text { Cycle } \\
\text { conjoncturel } \\
\text { positif de } \\
1991 \text { à } 1995\end{array}$ & $\begin{array}{c}\text { Cycle } \\
\text { conjoncturel } \\
\text { négatif en } \\
1991 \text { et } \\
\text { positif de } \\
1992 \text { à } 1995\end{array}$ & $\begin{array}{c}\text { Cycle } \\
\text { conjoncturel o } \\
\text { négatif en } \\
1991 \text { et } 1992 \\
\text { et positif de } \\
1993 \text { à } 1995\end{array}$ & $\begin{array}{c}\text { Cycle } \\
\text { conjoncturel } \\
\text { négatif en } \\
1991,1992 \\
1993 \text { et } \\
\text { positif en } \\
1994 \text { et } 1995\end{array}$ \\
\hline 1991 & 410290 & 393807 & 393807 & 393807 \\
\hline 1992 & 421502 & 421502 & 405019 & 405019 \\
\hline 1993 & 433391 & 433391 & 433391 & 416909 \\
\hline 1994 & 445959 & 445959 & 445959 & 445959 \\
\hline 1995 & 459204 & 459204 & 459204 & 459204 \\
\hline Total & 2170346 & 2153863 & 2137380 & 2120898 \\
\hline Moyenne & 434069 & 430773 & 427476 & 424180 \\
\hline $\begin{array}{l}\text { Pertes par } \\
\text { rapport à } \\
\text { l'hypothès. }\end{array}$ & $\mathrm{A}$ & 16483 & 32966 & 49448 \\
\hline
\end{tabular}

\section{TABLEAU 5}

Prévisions à court terme* (par mois) des chambres disponibles, des nuitées et des taux d'occupation à Montréal de septembre 1991 à août 1992

\begin{tabular}{lccr}
\hline Mois & Chambres & Nuitées & Taux** \\
\hline Septembre 1991 & 409450 & 297768 & 72.6 \\
Octobre 1991 & 422191 & 263882 & 62.4 \\
Novembre 1991 & 409121 & 207054 & 50.6 \\
Décembre 1991 & 421626 & 155834 & 36.9 \\
Janvier 1992 & 426481 & 174998 & 41.2 \\
Février 1992 & 384595 & 197209 & 51.4 \\
Mars 1992 & 424833 & 217226 & 49.9 \\
Avril 1992 & 413600 & 221289 & 53.9 \\
Mai 1992 & 427873 & 271353 & 63.4 \\
Juin 1992 & 415571 & 279319 & 66.7 \\
Juillet 1992 & 430037 & 259262 & 60.3 \\
Août 1992 & 431848 & 323086 & 75.4 \\
\hline
\end{tabular}

* Calculés à partir des méthodes X11 et Stepar à l'aide du logiciel SAS.

** Il s'agit des projections indépendantes; donc ici le taux d'occupation ne correspond pas à: (nuites/chambres) $\times 100$.

\section{TABLEAU 7}

Les prévisions* de la moyenne annuelle des nuitées dans les hôtels de Montréal, de 1991 à 1995, en fonction de quatre hypothẻses d'évolution de la conjoncture économique

\begin{tabular}{lllll}
\hline Hypothèses & A & B & C & D \\
Années
\end{tabular}

\section{Années}

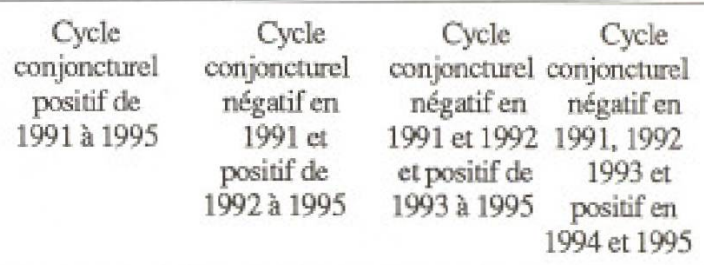

\begin{tabular}{lrrrr}
\hline 1991 & 274820 & 255040 & 255040 & 255040 \\
1992 & 279743 & 279743 & 259963 & 259963 \\
1993 & 284668 & 284688 & 284668 & 264888 \\
1994 & 289596 & 289596 & 289526 & 289596 \\
1995 & 294526 & 294526 & 294526 & 294526 \\
$\begin{array}{l}\text { Total } \\
\text { Moyenne }\end{array}$ & 284671 & 280714 & 276759 & 272803 \\
$\begin{array}{l}\text { Moy } \\
\text { Pertes par }\end{array}$ & & 1403573 & 1383793 & 1364013 \\
$\begin{array}{l}\text { rapport à } \\
\text { l'hypothèse A }\end{array}$ & & & & \\
\hline
\end{tabular}

* Il s'agit d'une tendance parabolique couplée à quatre possibilités d'évolution du cycle conjoncturel. 
évolueront avec des hauts et des bas! Ces prévisions mécaniques se calquent sur la situation économique d'ensemble qui n' ${ }^{\prime}$ est pas au beau fixe; la reprise tarde à se manifester, les indicateurs de l'expansion piétinent et donnent une image de stagnation généralisée.

\section{Les prévisions à moyen terme}

Les prévisions de la moyenne annuelle des chambres disponibles dans les hôtels de Montréal, de 1991 à 1995, sont présentées dans le tableau 6. Dans ce tableau, nous avons formulé quatre hypothèses:

1) la première hypothèse (A) suppose un cycle conjoncturel positif de 1991 à 1995 (la teprise démarre vraiment en janvier 1991);

2) la deuxième hypothèse (B) présume que le cycle conjoncturel sera négatif en 1991 et positif pour les années 1992 à 1995;

3) dans la troisième hypothèse (C), nous imaginons que le cycle conjoncturel est négatifen 1991 et 1992 et positifde 1993 à 1995;

4) enfin, la quatrième hypothèse (D), implique que le cycle conjoncturel soit mégatif de 1991 à 1993 et positif pour 1994 et 1995.

Dansle modèle $B$, et pour lescinq prochaines années, il y aurait, au total, une perte de 16 483 chambres, dans le modèle C une perte de 32966 chambres et dans le modele D, une perte de 49448 chambres (par rapport à l'hypothèse A).

Nous devons d'emblée rejeter l'hypothèse A car les moyennes des chambres disponibles de 1991, par rapport à 1990, indiquent que l'annee 1991 est une année négative en terme de conjoncture. Dans le cas des chambres disponibles, les seules hypothèses plausibles sont les hypothèses $\mathrm{B}$ et C. Dans le passé, lecycle conjoncturel négatif des chambres disponibles ne dépassait pas une année (sauf en 1985 et 1986), ce qui nous amène donc à rejeter aussi 1'hypothêse D. Retenir l"hypothèse C serait supposer que la récession actuelle (plus ou moins agonisante) serait semblable à celle que nous avons connue de 1981 à 1985: cela semble peu probable. L'hypothèse B est donc LA MEILLEURE
HYPOTHESE d'évolution des chambres disponibles de 1991 à 1995.

Nous avons effectué les mêmes hypothèses prévisionnelles pour les nuitées de 1991 à 1995 (voir le tableau 7). Par rapport à l'hypothèse A, les pertes seraient de 19780 nuitces pour 1 'hypothèse $B$, de 39560 nuitées pour l'hypothèse Cet de 59340 nuitées pour l'hypothèse D.

Dans le cas des nuitées, le cycle négatif a débuté en 1989; donc, en incluant 1991, ce cycle compterait trois années. De 1981 à 1985, on a observé une baisse des nuitées (un cycle négatif de près de cinq années).

Notre choix se limite aux hypothèses B et C. En retenant 1'hypothèse $\mathrm{D}_{\text {, }}$ on supposerait que le cycle négatif (pour les nuitées) sera semblable à celui des années 19811985 et durerait cinq années (de 1989 à 1993). La situation la plus probable est que l'expansion Economique présumée ne démarrera vraiment qu'à la fin de 1992; dans ces conditions, 1'hypothèse C apparaît comme LA MEILLEURE HYPOTHESE d'êvolution des nuitées de 1991 a 1995.

\section{Le bilan de santé de I'hôtellerie à Montréal}

Nous pouvons conclure que les chambres disponibles ont une évolution quasi-linéaire entre 1977 et 1990; cette évolution est toujours décalée par rapport à la courbe des nuitées. L'ajustement entre les deux variables se fait toujours avec un certain retard. Le taux d'accroissement annuel moyen des chambres disponibles de 1980 à 1990 est de $1,8 \%$.

Le taux d'accroissement annuel moyen des nuites est à peine de $1 \%$. Pour les nuitées, il faut souligner la forte influence du cycle conjoncturel; cette dépendance laisse peu de choix au plan des moyens car comment construire une politique anti-cyclique ayant des effets directs sur l'industrie hôtelière? Ii faut remarquer aussi que le cycle hôtelier semble précéder l'ensemble du cycle économique puisque les indices sont négatifs dès 1988-1989.

Les mouvements saisonniers varient très peu sur une décennie; ce sont pratiquement des invariants du système hôtelier. Le confort climatique ${ }^{(6)}$ est une contrainte incontournable pour la prochaine décennie; cettecontrainteclimatique pèse lourdement sur tous les développements futurs.

L'êtude des mouvements irréguliers dans les séries temporelles étudiées (chambrées disponibles, nuitếes et taux d'occupation) semble peu probante. Ces mouvements, calculés à partir d'un indice $100^{\circ}$, ne dépassent pas $\pm 3,7 \%$ en moyenne dans les quatorze dernières années (1977 à 1990); ces mouvements semblent donc mineurs dans l'analyse de l'éwolution de ces séries.

Donc, un bilan de santé «ni chair ni poissons, uni rose ni noirs, où l'on doit doser les qualificatifs et tenir compte de l'évolution parcourue. Dans un pays comme le nôttre où, malgré tout ce qui s'écrit sur le sujet, les atouts touristiques ne sont ni nombreux ni évidents, l'industrie hôtelière semble bien se porter malgré des forces contraires. Ces forces contrairesce sont: le déclin relatif de Montréal, un faible niveau de compétitivité, le manque de données de base sur l'hôtellerie, la faible placedu secteur hôtelier dans les politiques touristiques. Donc, beaucoup de boulot pour la prochaine décennie...

\section{Notes et références}

I1) Voif a ce sujet deus livres recents: R. Bar On, Travel and Tourism Data, Oryx Press, Fhoenix, 1989 et F. Witt, Modeling and Foracasting Domand in Tourism, Academic Ptess, London, 1991.

(2) M. David et J."C. Michaud, La prévision: approche empirique d'une méthode statistique. Masson, Paris, 1969, p. 22

(3) Voir la descriotion détallée de cette apcroche dans P. Bodsonet J. Stafford, w Provisions à court terme pour l'hätellarie â Montréali, da ns Túoros. vol. $8, n \circ 2$, juillet 1989 , po, 3-5.

(4) Voira ce sujet G. Melard Máthodes de prévision a court terme, Éditions de l'Universite de Bruxelles, Bruxelles, 1990.

(5) Pour une definition plus presise woir B. Py, Statistique descriptive, Économica, Paris, 1987. p. 238.

(6) Voir à ce sujet لي.P. Besanoenot, climat ot tourismo, Masson, Paris, 1990

(7) Indices irréguliers calculés par l'algonthme $X 11$ de SAS. 